\title{
Correction to: Multi-center, randomized, double-blind, placebocontrolled study of quetiapine extended-release formulation in Japanese patients with bipolar depression
}

\author{
Mitsukuni Murasaki ${ }^{1}$ - Tsukasa Koyama ${ }^{2} \cdot$ Shigenobu Kanba $^{3} \cdot$ Masahiro Takeuchi $^{4} \cdot$ Yuriko Shimizu $^{5} \cdot$ Eri Arita $^{6}$. \\ Kentaro Kuroishi $^{7} \cdot$ Masahiro Takeuchi $^{8} \cdot$ Shinya Kamei $^{5}$
}

Published online: 17 September 2018

(C) Springer-Verlag GmbH Germany, part of Springer Nature 2018

\section{Correction to: Psychopharmacology \\ https://doi.org/10.1007/s00213-018-4977-6}

The original version of this article contained a mistake in

Table 5. Correct version is presented here.

The online version of the original article can be found at https://doi.org/ 10.1007/s00213-018-4977-6

Shinya Kamei

shinya.kamei@astellas.com

Mitsukuni Murasaki

murasaki@i-cnsp.com

Tsukasa Koyama

koyama@ohyachi-hp.or.jp

Shigenobu Kanba

skanba@npsych.med.kyushu-u.ac.jp

Masahiro Takeuchi

takeuchim@pharm.kitasato-u.ac.jp

Yuriko Shimizu

yuriko.shimizu@astellas.com

Eri Arita

eri.arita@astellas.com

Kentaro Kuroishi

kentarou.kuroishi@astellas.com

Masahiro Takeuchi

masahiro-takeuchi@astellas.com
Institute of CNS Pharmacology, 3-14-20 Sagamiohno, Minami-ku, Sagamihara, Kanagawa 252-0303, Japan

2 Ohyachi Hospital, Clinical Research Center, 5-7-10 Ohyachi-higashi, Atsubetsu-ku, Sapporo, Hokkaido 004-0041, Japan

3 Department of Neuropsychiatry, Graduate School of Medical Sciences, Kyushu University, 3-1-1 Maidashi, Higashi-ku, Fukuoka 812-8582, Japan

4 Department of Clinical Medicine, School of Pharmacy, Kitasato University, 5-9-1 Shirokane, Minato-ku, Tokyo 108-8641, Japan

5 Japan/Asia Clinical Development 2, Astellas Pharma Inc., 2-5-1 Nihonbashi-Honcho, Chuo-ku, Tokyo 103-8411, Japan

6 Global Clinical Science, Astellas Pharma Europe B.V, Sylviusweg 62, PO Box 344, 2300 AH Leiden, The Netherlands

7 Japan-Asia Data Science, Astellas Pharma Inc., 2-5-1 Nihonbashi-Honcho, Chuo-ku, Tokyo 103-8411, Japan

8 Astellas Pharma Global Development, Inc., 1 Astellas Way, Northbrook, IL 60062, USA 


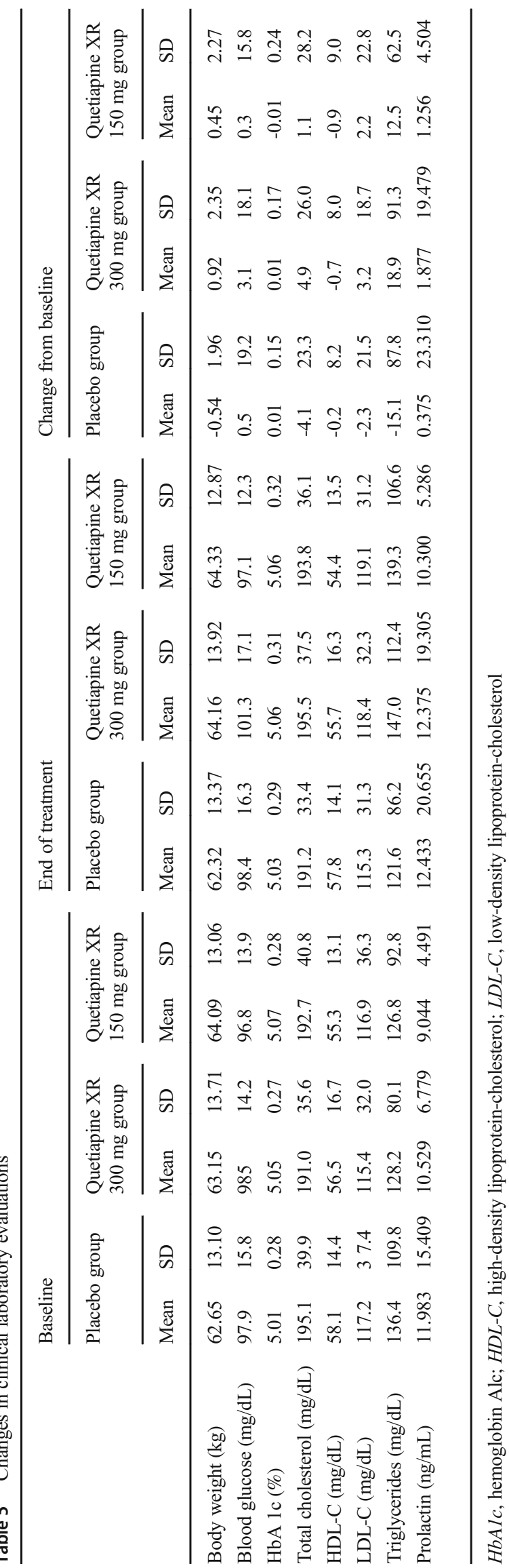

